\title{
The Parkinson's disease sleep scale: a new instrument for assessing sleep and nocturnal disability in Parkinson's disease
}

\author{
K R Chaudhuri, S Pal, A DiMarco, C Whately-Smith, K Bridgman, R Mathew, \\ F R Pezzela, A Forbes, B Högl, C Trenkwalder
}

See end of article for authors' affiliations

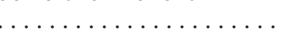

Correspondence to: Dr K R Chaudhuri, Department of Neurology, King's College Hospital, Denmark Hill, London, SE5 9RS:

ray.chaudhuri@

kingshc.nhs.uk

Received 30 April 2002 In revised form 19 July 2002

Accepted 20 August 2002
Background: No formal instruments are available for quantifying sleep problems in Parkinson's disease.

Objective: To develop a new sleep scale to quantify the various aspects of nocturnal sleep problems in Parkinson's disease, which may occur in up to $96 \%$ of affected individuals.

Methods: Employing a multidisciplinary team approach, a visual analogue scale was devised addressing 15 commonly reported symptoms associated with sleep disturbance in Parkinson's disease-the Parkinson's disease sleep scale (PDSS). In all, 143 patients with Parkinson's disease completed the PDSS, covering the entire spectrum of disease from newly diagnosed to advanced stage. As controls, 137 age healthy matched subjects also completed the scale. Test-retest reliability was assessed in a subgroup of subjects. The Epworth sleepiness scale was also satisfactorily completed by 103 of the patients with Parkinson's disease.

Results: PDSS scores in the Parkinson group were significantly different from the healthy controls. Patients with advanced Parkinson's disease had impaired scores compared with early/moderate disease. Individual items of the scale showed good discriminatory power between Parkinson's disease and healthy controls. Relevant items of the PDSS correlated with excessive daytime sleepiness. The scale showed robust test-retest reliability.

Conclusions: This appears to be the first description of a simple bedside screening instrument for evaluation of sleep disturbances in Parkinson's disease. A combination of subitems may help identify specific aspects of sleep disturbance, which in turn may help target treatment.
S eep disturbances occur in up to $96 \%$ of patients with Parkinson's disease and appear to arise from a combination of neurochemical and neurodegenerative changes in central sleep regulatory centres such as the forebrain, thalamus, and midbrain dopamine neurones. ${ }^{1-3}$ Sleep disturbances and sleepiness are variably manifested by excessive daytime naps, hypoactivity response to dopaminergic stimulation, and alteration in dopaminergic transmission in target organs. ${ }^{34}$ These factors underline the importance of the dopaminergic system in the maintenance of normal sleep, and recent surveys have revealed the importance of sleep disturbance in determining the quality of life of patients with Parkinson's disease. In this disorder, studies of sleep architecture show alterations in stages 3, 4, and rapid eye movement (REM) sleep. ${ }^{56}$ However, motor and related neuropsychiatric symptoms are also important causes of sleep disturbance in Parkinson's disease. ${ }^{78}$

Currently, no formal instruments are available for quantifying the various aspects of nocturnal sleep problems in Parkinson's disease. Scales widely employed in clinical practiceincluding the Epworth sleepiness scale (ESS) and the Pittsburgh sleep quality index (PSQI) - do not systematically address and quantify the different aspects of sleep disturbance in Parkinson's disease. ' Lang and colleagues have used a modified version of the ESS (MESS) and reported that the ESS has poor sensitivity for predicting sudden sleep onset during driving, while Arnulf et al have reported that excessive daytime sleepiness as measured by the ESS is not dependent on nocturnal sleep disturbances in Parkinson's disease. ${ }^{40}$ The unified Parkinson's disease rating scale (UPDRS) contains only one question related to sleep problems, and the newly validated Parkinson's disease quality of life scale (PDQ39) is also limited in terms of questions related to sleep. ${ }^{11}$ Thus a validated, simple to use, bedside clinical instrument to provide a semiquantitative assessment of the multifactorial nature of sleep problems in Parkinson's disease would be useful.

Employing a multicentred, multidisciplinary team approach (involving a neurologist, a neurophysiologist, a psychologist, and a Parkinson's disease nurse specialist), and with collaboration from colleagues in Germany (CT, BH) and Italy (FRP), we have devised a tool for comprehensive assessment of the symptoms contributing to sleep disturbance in Parkinson's disease. We have termed this instrument the Parkinson's disease sleep scale (PDSS) ${ }^{712}$ (fig l). The scale is currently available in four languages and has been used successfully within our group to evaluate the frequency and specific nature of sleep problems in Parkinson's disease and to help treat them.

The PDSS is a visual analogue scale addressing 15 commonly reported symptoms associated with sleep disturbance (fig 1). The 15 items chosen are based on audit of our experiences in relation to factors causing sleep disturbances in over 800 patients with Parkinson's disease attending outpatient hospital clinics (between 1996 and 2000), in addition to the reports of caregivers. In this study we describe the validation and clinical use of the PDSS in the management of Parkinson's disease.

Abbreviations: ESS, Epworth sleepiness scale; MESS, modified Epworth sleepiness scale; PDSS, Parkinson's disease sleep scale; PSQI, Pittsburgh sleep quality index; UPDRS, unified Parkinson's disease rating scale 


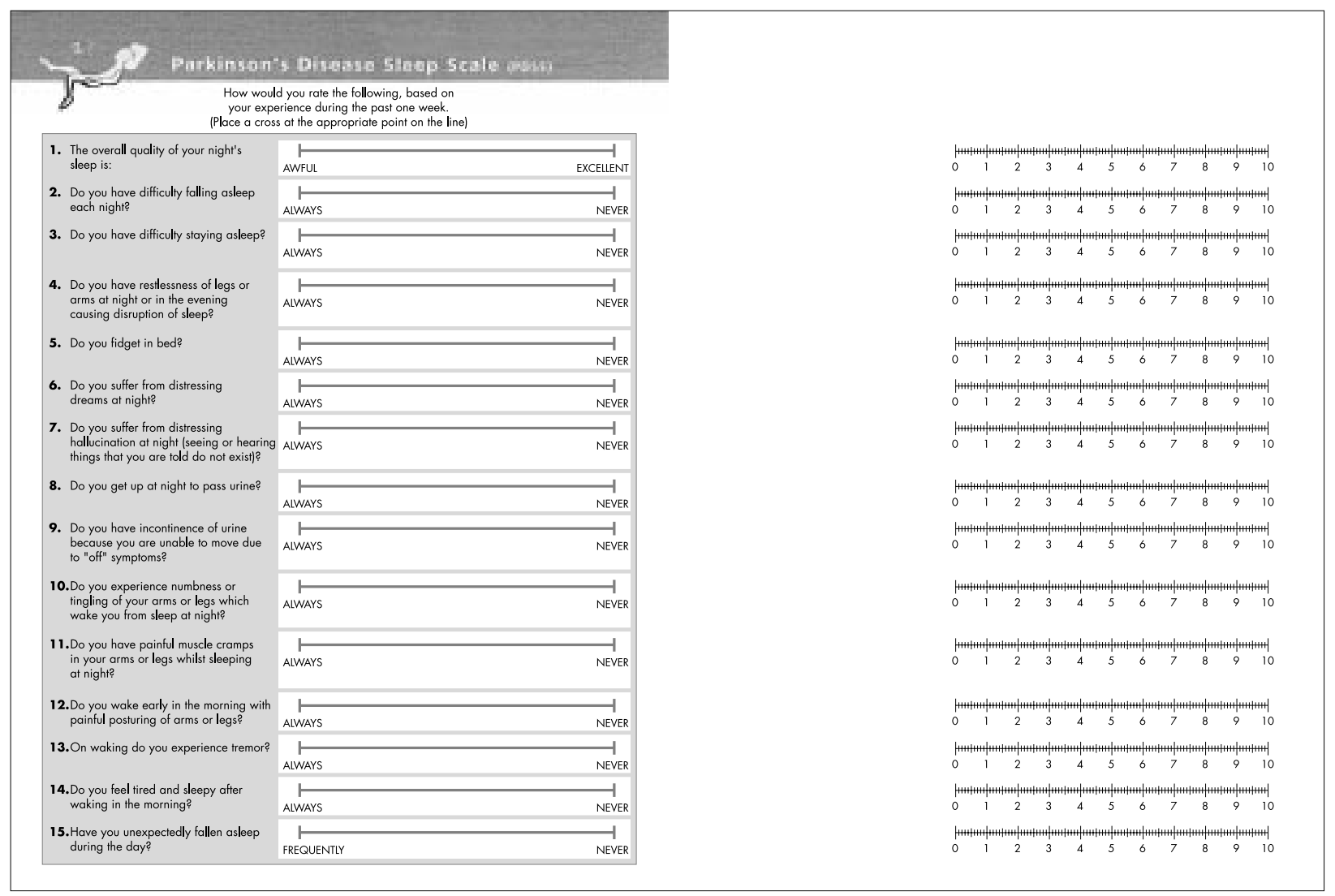

Figure 1 Right panel: the actual scale as presented to patients, who are asked to mark their responses according to severity by placing a cross mark on the $10 \mathrm{~cm}$ line. The $\mathrm{mm}$ scale which is printed on a transparency (shown on the left panel) is then applied on the $10 \mathrm{~cm}$ lines to measure the responses in decimal figures; 10 represents excellent/never responses; 0 represents the worst score.

\section{METHODS}

\section{The PDSS}

Items of the PDSS address the following:

- overall quality of night's sleep (item 1);

- sleep onset and maintenance insomnia (items 2 and 3);

- nocturnal restlessness (items 4 and 5);

- nocturnal psychosis (items 6 and 7)

- nocturia (items 8 and 9);

- nocturnal motor symptoms (items 10-13);

- sleep refreshment (item 14);

- daytime dozing (item 15).

\section{Completion of the PDSS}

Patients, or caregivers (by proxy), ${ }^{13}$ completed the PDSS, based on their experiences in the past week. Patients were asked to fill in the PDSS either in the consultation room or at home; involvement of the caregiver was encouraged. The severity of symptoms was reported by marking a cross along a $10 \mathrm{~cm}$ line (labelled from worst to best state). Responses were quantified by measuring the distance along each line to the intersection with the cross in centimetres, to the nearest $0.1 \mathrm{~cm}$. Thus scores for each item range from 0 (symptom severe and always experienced) to 10 (symptom-free). The maximum cumulative score for the PDSS is 150 (patient is free of all symptoms).

Evaluation and calculation of the data were done by SP, AD, RM, and FRP. As the PDSS is employed as part of routine clinical practice and audit in the outpatient clinic assessment of patients with Parkinson's disease, the institutional ethics committee agreed that specific ethical approval was not required for this study.

\section{Subjects}

In all, 280 adult subjects completed the PDSS, 143 patients with Parkinson's disease and 137 aged matched healthy controls.

\section{Parkinson's disease patients}

The 143 patients with Parkinson's disease completed the PDSS as part of local routine clinical practice, while attending outpatient clinics at King's College and Lewisham hospitals. There were 89 men (62\%) and 54 women. Their mean (SD) age was 67.0 years (range 38 to 89 ), and the duration of their disease was 6.0 (5.1) years (range 1 to 26). The Hoehn and Yahr score was $2.7(0.7)$, range 1 to 4 . Subjects included the entire spectrum of Parkinson's disease from newly diagnosed to treated patients in the advanced stages. Those with clinical features suggestive of parkinsonian syndromes due to multiple system atrophy, progressive supranuclear palsy, or Lewy body dementia were excluded. We also excluded subjects with cognitive impairment who were unable to complete the PDSS.

Demographic details and antiparkinsonian, sedative, and antidepressant drugs prescribed for the patients were noted.

\section{Controls}

The 137 healthy age matched controls who completed the PDSS were principally hospital employees and relatives, with no known concomitant medical conditions (non-obese with no past medical history of significant neurological or respiratory disorders). There were 61 men $(44 \%)$ and 76 women. Their mean (SD) age was 65.6 (11.7) years (range 35 to 93).

\section{Test-retest reliability and repeatability of scale}

To investigate its repeatability, 15 patients with Parkinson's disease completed the PDSS on two separate occasions with a 


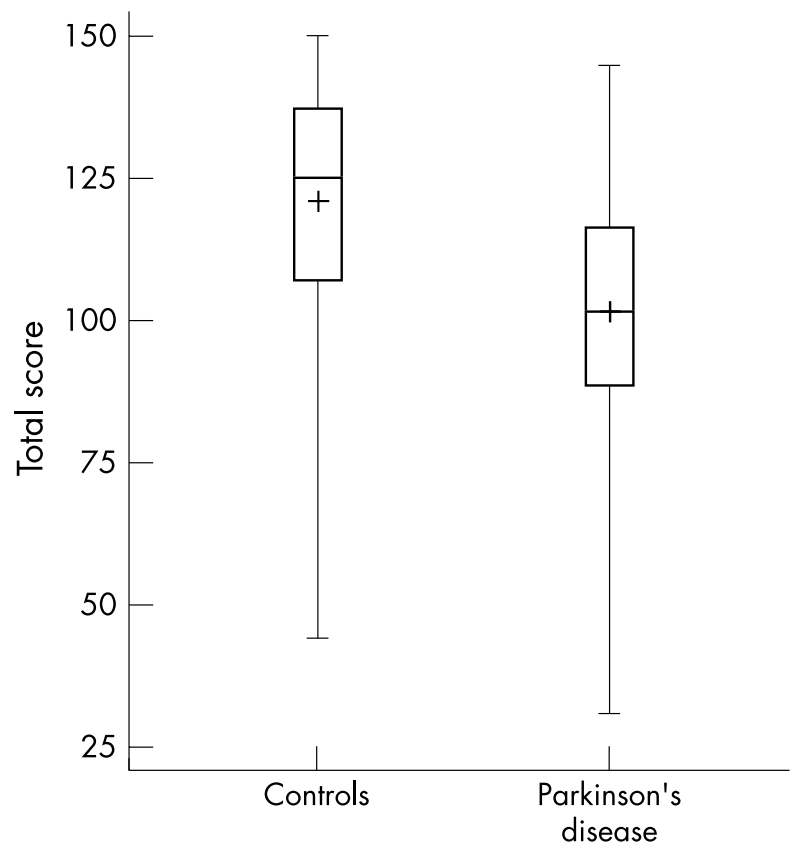

Figure 2 Box plot representation of total PDSS (Parkinson's disease sleep scale) scores obtained by patients with Parkinson's disease (PD) and controls. Square denotes the mean values; lines denote the median values; box denotes specific interquartile range. Maximum and minimum scores are indicated by the upper and lower markers, respectively.

one to two week interval, under standardised conditions with the same health care professional administering the scale on each occasion.

\section{Assessment of impact of nocturnal symptoms on excessive daytime sleepiness}

To investigate the impact of nocturnal disabilities on excessive daytime sleepiness, the ESS was administered to the Parkinson's disease group, and 103 patients completed the scale satisfactorily during the same visit as the PDSS (66 men (64\%), 37 women; mean age 66.9 (9.8) years; mean duration of disease 5.6 (4.7) years; mean Hoehn and Yahr score $2.7(0.7))$.

\section{Statistical methods}

Simple descriptive statistics were used to summarise the responses to each item and the total, and in particular to identify "ceiling" and "floor" responses-respectively, the maximum and minimum possible scores. A high number of either indicates that the scale may not be asking the right questions and has no discriminatory power. The correlation coefficients between each pair of items were calculated to investigate the interrelations. Any particularly high correlation would indicate possible redundancy of items if they were effectively recording the same thing.

For test-retest reliability, the intraclass correlation coefficient (ICC) was calculated using the estimated between and within subjects variances derived from the analysis of variance.$^{14}$ This was done for the total scores and for the individual items, to see whether there were problems in relation to reproducibility associated with any items individually. To demonstrate reasonable repeatability, an ICC of at least 0.7 is desirable.

To assess the sensitivity of the scale in distinguishing between patients with Parkinson's disease and controls, the total score was compared between groups using analysis of variance. Classifying the Parkinson's disease patients according to their Hoehn and Yahr score extended this: those scoring

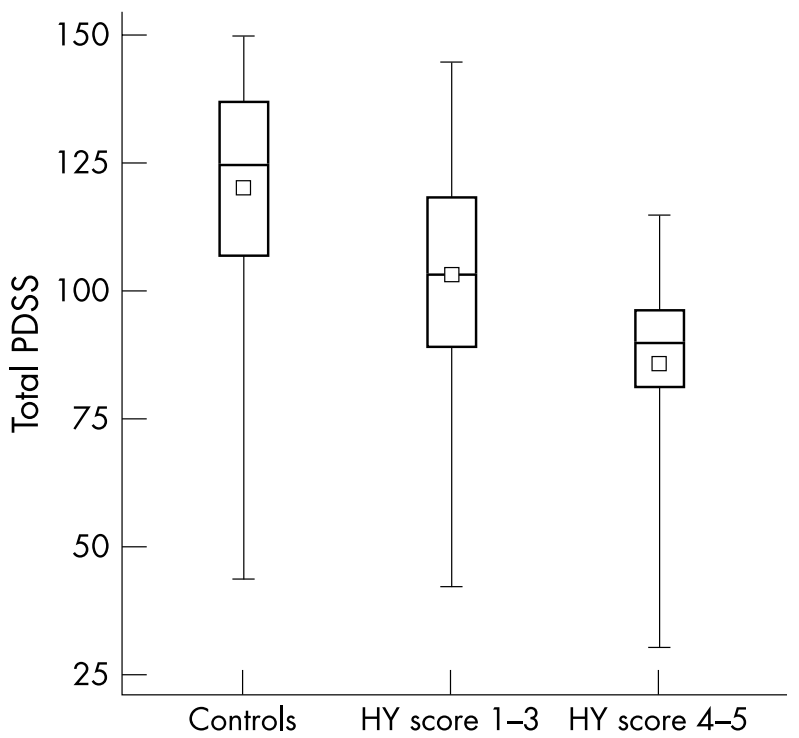

Figure 3 Box plot representation of total PDSS (Parkinson's disease sleep scale) scores obtained by patients with Parkinson's disease (PD) and controls. PD patients are grouped into early/moderate PD (Hoehn and Yahr scores between 1 and 3 ) and advanced PD (4 to 5). Square denotes the mean values; lines denote the median values; box denotes specific interquartile range. Maximum and minimum scores are indicated by the upper and lower markers, respectively.

between 1 and 3 were classified as early/moderate Parkinson's disease and those scoring 4 or 5 were classified as advanced Parkinson's disease. Analysis of variance was again used to see if there were differences. The mean difference between the groups and the associated 95\% confidence intervals were derived from the analysis. The confidence intervals for the difference between each group of Parkinson's disease patients and controls were derived using Dunnett's method, which allows for repeated testing against the same group of controls. The distribution of the total scores was depicted graphically using a box plot, which shows the extreme scores, the mean, the median, and the interquartile range. In addition, the means for the controls and the Parkinson's disease patients were shown for each item.

The scores for each item for the two groups were compared using unpaired $t$ tests. In order to adjust for the multiple testing which arises out of testing related data from the same patients, the stepdown Bonferroni method presented by Holm $^{15}$ was used to adjust the $p$ values to avoid problems of attaining false significance.

To investigate criterion validity, the ESS was administered to the patients simultaneously. As the ESS addresses sleepiness during the day, the only relevant item on the PDSS was item 15. To investigate the relation between these two scales, the data were plotted against each other for the 103 patients who had satisfactorily completed both the ESS and the PDSS.

\section{RESULTS}

The correlation coefficients between the items were all positive, indicating that scores for all items lay in the same direction. Correlations within the control group were generally higher than within the Parkinson group, mainly because of the larger number of subjects who scored 10 (best score) on each item. Among the patients with Parkinson's disease, the highest correlation was seen between items 1 (overall quality of sleep) and 3 (difficulty staying asleep), with a value of 0.70 . The only other pair of items to achieve a correlation of 0.5 or above was items 4 and 5 (restlessness of arms and legs, fidgeting). 
Table 1 Probability values for each item of the PDSS comparing scores of patients with Parkinson's disease with those of controls

\begin{tabular}{lll}
\hline Variable & Contrast & $\begin{array}{l}\text { Stepdown Bonferroni } \\
\text { p value }\end{array}$ \\
\hline var1 & PD $v$ control & 0.0573 \\
var2 & PD $v$ control & 0.5804 \\
var3 & PD $v$ control & 0.0061 \\
var4 & PD $v$ control & 0.0004 \\
var5 & PD $v$ control & 0.0045 \\
var6 & PD $v$ control & $<0.0001$ \\
var7 & PD $v$ control & $<0.0001$ \\
var8 & PD $v$ control & $<0.0001$ \\
var9 & PD $v$ control & $<0.0001$ \\
var10 & PD $v$ control & 0.0004 \\
var1 1 & PD $v$ control & $<0.0001$ \\
var12 & PD $v$ control & 0.0070 \\
var13 & PD $v$ control & $<0.0001$ \\
var14 & PD $v$ control & 0.0573 \\
var15 & PD $v$ control & $<0.0001$ \\
\hline
\end{tabular}

PD, Parkinson's disease; PDSS, Parkinson's disease sleep scale.

Analysis of variance comparing total PDSS scores between Parkinson's disease and controls showed a highly significant difference $(p<0.0001)$ (fig 2$)$. Furthermore, PDSS scores were markedly different between early/moderate Parkinson's disease $(\mathrm{HY}=1-3)$, advanced disease $(\mathrm{HY}=4-5)$, and control subjects $(p=0.007)$ (fig 3$)$. Patients with advanced disease had substantially lower scores than those with early/moderate disease, at 86 (21) $v 103$ (21), respectively. The mean difference between controls and patients with early/ moderate Parkinson's disease was 17.7 (95\% confidence interval, 11.8 to 23.5); the mean difference between controls and patients with advanced Parkinson's disease was 34.7 (20.9 to 48.4). These differences show that the scale provides good discriminative power in differentiating between the sleep problems of patients with advanced Parkinson's disease and those of patients with early Parkinson's disease.

The summary of responses to each item and the total score for the Parkinson group and controls is shown in a profile format in fig 4. Only one item was missing for one Parkinson's disease patient: item 9. There were highly significant differences between the patients and controls for most items except item 2 (difficulty in falling asleep) (table 1 ). The difference for items 1 (overall quality of nocturnal sleep) and 14 (sleep refreshment) was borderline $(\mathrm{p}=0.0573)$. The Parkinson group scored approximately 1.5 points lower than the

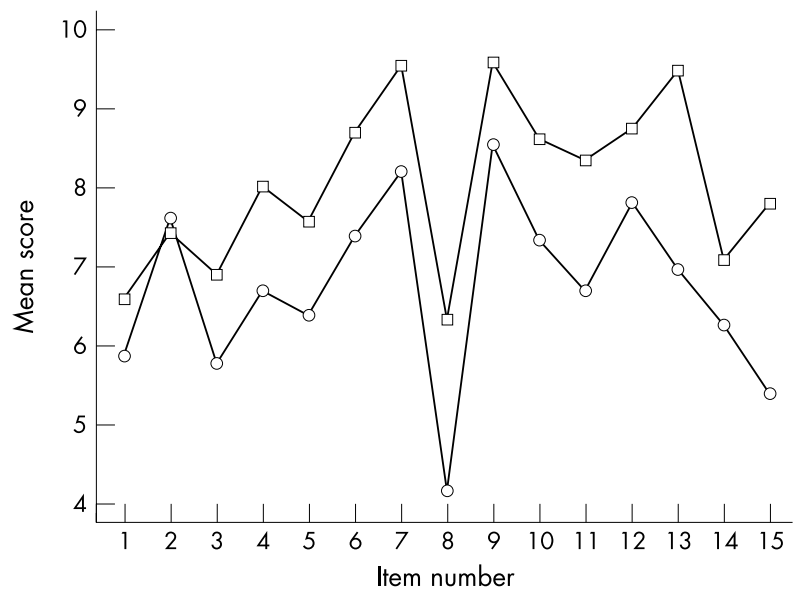

Figure 4 Profile of mean PDSS (Parkinson's disease sleep scale) scores of each item between Parkinson's disease (PD) and controls (C). All differences were highly significant ( $p<0.01$; table 2$)$ except item $2(p=0.58)$, where the differences were non-significant and without a trend, and items 1 and 14, where the differences were borderline $(p=0.0573)$

controls (table 2). Profiles were similar between the two groups (fig 4), with item 8 (nocturia) scores being lowest in both groups. The number of controls scoring 10 for any item was considerably higher than the number of patients scoring 10 (table 2). Generally the incidence of "floor" and "ceiling" responses was low among the patients.

When item 15 (related to daytime naps) was correlated with the ESS, a strong relation was found, with a correlation coefficient of -0.59 . This indicates that high scores on the PDSS are associated with low scores on the ESS. Figure 5 illustrates the relation between the two measures.

The results of the repeatability analysis are summarised in table 3 . The repeatability estimated using the intraclass correlation coefficient (ICC) was 0.94 for the total score, which is high. The lower $95 \%$ confidence limit for this estimate is 0.89 , which confirms good reliability for this group of patients and controls. The repeatability for the individual items in the questionnaire was also high. The within patient variability for the scores on items 4,8 , and 11 was particularly high. Some items did, however, show a greater spread of responses, especially items 1, 6, and 14. Item 8 displayed a bimodal response, with patients either scoring very high or very low.

Table 2 PDSS total score and individual items

\begin{tabular}{|c|c|c|c|c|c|c|c|c|}
\hline \multirow[b]{2}{*}{ Item } & \multicolumn{4}{|c|}{ Controls $(n=137)$} & \multicolumn{4}{|c|}{ Parkinson's disease $(n=143)$} \\
\hline & Mean & $S D$ & $\begin{array}{l}\text { Scoring } 0 \\
\text { (n) }\end{array}$ & $\begin{array}{l}\text { Scoring } \\
\max (n)\end{array}$ & Mean & SD & $\begin{array}{l}\text { Scoring } 0 \\
\text { (n) }\end{array}$ & $\begin{array}{l}\text { Scoring } \\
\max (n)\end{array}$ \\
\hline Total score & 120.7 & 21.0 & 0 & 2 & 101.1 & 21.7 & 0 & 0 \\
\hline Item 1 & 6.6 & 2.6 & 0 & 19 & 5.9 & 2.7 & 0 & 5 \\
\hline Item 2 & 7.4 & 2.8 & 1 & 35 & 7.6 & 2.5 & 1 & 10 \\
\hline Item 3 & 6.9 & 2.9 & 0 & 27 & 5.8 & 2.9 & 1 & 2 \\
\hline Item 4 & 8.0 & 2.7 & 1 & 50 & 6.7 & 2.7 & 0 & 6 \\
\hline Item 5 & 7.6 & 2.8 & 1 & 41 & 6.4 & 2.9 & 0 & 8 \\
\hline Item 6 & 8.7 & 1.7 & 0 & 44 & 7.4 & 2.6 & 1 & 9 \\
\hline Item 7 & 9.5 & 1.0 & 0 & 87 & 8.2 & 2.2 & 0 & 12 \\
\hline Item 8 & 6.3 & 3.2 & 1 & 20 & 4.2 & 2.8 & 3 & 3 \\
\hline Item 9 & 9.6 & 1.3 & 1 & 94 & 8.5 & 1.8 & 0 & 21 \\
\hline Item 10 & 8.6 & 2.5 & 2 & 71 & 7.3 & 2.6 & 0 & 15 \\
\hline Item 11 & 8.4 & 2.4 & 1 & 59 & 6.7 & 2.8 & 0 & 8 \\
\hline Item 12 & 8.8 & 2.5 & 1 & 79 & 7.8 & 2.5 & 1 & 11 \\
\hline Item 13 & 9.5 & 1.5 & 1 & 90 & 7.0 & 2.8 & 3 & 6 \\
\hline Item 14 & 7.1 & 2.9 & 2 & 30 & 6.3 & 2.9 & 3 & 3 \\
\hline Item 15 & 7.8 & 2.6 & 1 & 43 & 5.4 & 3.0 & 6 & 5 \\
\hline
\end{tabular}




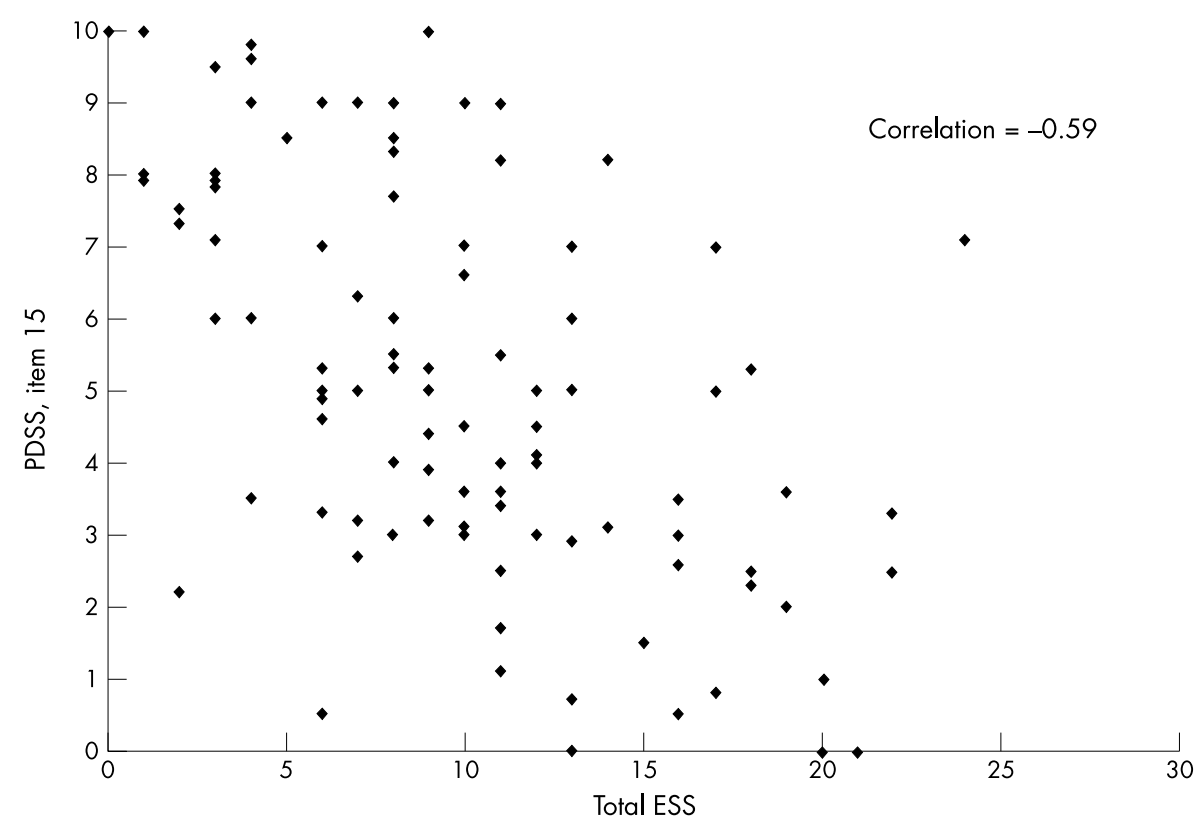

Figure 5 Scatterplot of item 15 (unexpectedly falling asleep during the day) against scores on the Epworth sleepiness scale (ESS). These data yield a significant correlation coefficient of -0.59 .

Table 3 Estimates of variance components and reliability based on repeatability analysis

\begin{tabular}{llll}
\hline Item & $\sigma_{\mathrm{s}}^{2}$ & $\sigma_{\mathrm{e}}^{2}$ & ICC \\
\hline Total & $\mathbf{7 4 4 . 3 8}$ & $\mathbf{4 5 . 9 7}$ & $\mathbf{0 . 9 4}$ \\
Item 1 & 5.12 & 0.56 & 0.90 \\
Item 2 & 2.50 & 1.45 & 0.61 \\
Item 3 & 6.03 & 0.96 & 0.86 \\
Item 4 & 10.04 & 0.19 & 0.98 \\
Item 5 & 6.63 & 0.31 & 0.96 \\
Item 6 & 5.60 & 0.49 & 0.92 \\
Item 7 & 3.01 & 0.26 & 0.92 \\
Item 8 & 13.61 & 1.59 & 0.89 \\
Item 9 & 8.78 & 0.12 & 0.99 \\
Item 10 & 3.38 & 1.76 & 0.66 \\
Item 11 & 11.33 & 1.28 & 0.89 \\
Item 12 & 8.61 & 0.81 & 0.91 \\
Item 13 & 9.57 & 2.16 & 0.82 \\
Item 14 & 7.03 & 1.06 & 0.87 \\
Item 15 & 5.25 & 3.12 & 0.62 \\
\hline
\end{tabular}

ICC, intraclass correlation; $\sigma_{\mathrm{e}}^{2}$, within patient variability; $\sigma_{\mathrm{s}}^{2}$, between patient variability.

\section{DISCUSSION}

This study shows that the PDSS is easy to use and is a reliable instrument for measuring sleep disturbances in Parkinson's disease. The PDSS scores were highly significantly different between healthy age matched controls and patients with Parkinson's disease. The patients with advanced Parkinson's disease also had significantly impaired PDSS scores compared with patients with early/moderate disease. Individual items showed good discriminatory power between Parkinson's disease subjects and healthy controls, and we found that poor scores on item 15 of the PDSS correlated with excessive daytime sleepiness measured with the ESS. The scale showed robust test-retest reliability.

To our knowledge this is the first description of a bedside instrument designed to assess the multifactorial nature of sleep disturbances in Parkinson's disease. Sleep disturbances are believed to occur in over $90 \%$ patients with this disorder at some stage, yet currently the UPDRS has only one question aimed at establishing sleep problems. ${ }^{11}$ Additional advantages of this visual analogue scale include its ease of administration and its ability to provide a quantitative measure of symptoms contributing to sleep disturbance. Potential applications of the scale in clinical practice merit further discussion, as follows.

\section{The need for an instrument such as the PDSS}

Work from our own group and others indicates that the aetiology of nocturnal disabilities in Parkinson's disease is multifactorial and that nocturnal motor symptoms are of importance. ${ }^{3} 81617$ Traditionally, sleep assessments in Parkinson's disease have taken the form of pure subjective questioning or, in some cases, measurement of sleep architecture. These techniques, however, do not provide a holistic assessment of the night time problems of sufferers from Parkinson's disease. It can be argued-given the importance of sleep function in this disease - that assessment of night time problems requires a specific instrument similar to the UPDRS. The PDSS aims to provide this. Some currently available tools, including the ESS, ${ }^{9}$ only address single items such as excessive daytime sleepiness and are thus not comprehensive. In a study of excessive daytime sleepiness and its potential relation to sudden onset sleep in 638 patients with Parkinson's disease, Lang et al recently concluded that the ESS has poor sensitivity for predicting falling asleep while driving, and may not be appropriate for assessing susceptibility to unintended sleep episodes. ${ }^{4}$ Other studies have indicated that ESS scores do not correlate significantly with multiple sleep latency test scores, thought to be the gold standard for measuring sleep, and especially REM sleep latency during the daytime. ${ }^{17}$ Thus we feel the PDSS may offer a more practical and relevant way of assessing sleep disruption in Parkinson's disease.

\section{Interpretation and use of the PDSS}

Sensitivity analysis showed that the PDSS successfully discriminated between healthy subjects and patients with Parkinson's disease in 13 of the 15 items, and the means of these item scores for the Parkinson group were at least 1.5 points lower than for the control group. While there were no significant differences between the patients and the controls on item 2 (sleep onset insomnia), there was a highly significant difference in item 3, suggesting that sleep maintenance insomnia is Parkinson's disease related. The lower PDSS scores obtained by the Parkinson group is consistent with the observation that the sleep disturbances measured here are 
more common in Parkinson's disease than in healthy controls. Furthermore, significant differences in PDSS scores were identified between early and advanced disease states, suggesting that advancing disease is more likely to predispose to sleep disruption. Worsening of scores in advanced Parkinson's disease was seen across all 15 items of the PDSS.

Thus the PDSS allows sensitive differentiation of the specific factors contributing to sleep disruption in Parkinson's disease, emphasising its potential value in targeting the most appropriate treatment for nocturnal symptoms in this condition. Additionally, specific symptoms can be identified by combining items such as 4 and 5 for nocturnal restlessness, 10 to 13 for nocturnal off periods, and 14 and 15 for daytime sleepiness, and thus help target the treatment. Indeed, from a combination of these subitems we have recently reported a syndrome of nocturnal restlessness similar to restless legs syndrome causing sleep disruption in Parkinson's disease. ${ }^{18}$ However, we accept that detailed assessment of complex sleep architecture requires polysomnography.

From the data in table 1 it can be seen that the standard deviations for the PDSS scores are reasonably constant across all items. Table 2 shows the numbers of patients with Parkinson's disease scoring maximum and minimum values for each item. This is important information for a new scale, because if there is a large number of patients scoring the maximum or minimum values the scale has limited usefulness. The data in table 2 show that relatively few patients achieved the maximum score of 10, in marked contrast to the control group, where the proportions scoring 10 were considerably higher.

The clinical usefulness of a scale such as the PDSS is exemplified by previous work from our own group and others showing that sustained dopaminergic stimulation at night is associated with improved subjective reports of sleep in Parkinson's disease, although sleep architecture may be unaltered. ${ }^{19-24}$ Furthermore, a recent report by Arnulf et al suggests that continuous nocturnal motor stimulation achieved by subthalamic stimulation also improves sleep architecture. $^{25}$

\section{Administration and repeatability of the PDSS}

We found the scale easy to administer and to comprehend. It can be completed by patient or caregiver with occasional proxy, as is commonplace with most scales employed in chronic neurodegenerative disorders. ${ }^{13}$ There was high intrapatient and interpatient reliability. The time interval between the two administrations (during test-retest evaluation) was relatively short (one to two weeks) to try to ensure stable conditions; however, we do not feel that bias caused by remembering previous scores was likely to have been introduced. The visual analogue system is advantageous in this respect.

\section{Excessive daytime sleepiness and PDSS scores in Parkinson's disease}

Excessive daytime sleepiness and unintended sleep episodes are increasingly being associated with motor vehicle and occupational accidents, impaired work performance, and possibly reduced quality of life. ${ }^{26}{ }^{27}$ In Parkinson's disease, this issue has received much attention lately, owing to controversy surrounding a report by Frucht et al of "sleep attacks," or unintended sleep episodes, leading to road traffic accidents in nine patients with Parkinson's disease taking non-ergot dopamine agonists. ${ }^{28}$ Rye and colleagues, however, have suggested that there is increased arousal and paradoxical alertness in patients with Parkinson's disease complaining of poor sleep. ${ }^{29}$ Thus the impact of nocturnal sleep disruption on excessive daytime sleepiness in Parkinson's disease is far from clear, and several reports have suggested the need for controlled studies addressing this issue. ${ }^{30-33}$ Our study indicates that poor PDSS scores, and in particular poor scores on item 15, are correlated strongly with high scores on the ESS. This is consistent with subjective reporting of patients who had poor nocturnal sleep and felt tired and sleepy during the daytime.

\section{Limitations of the PDSS}

There are limitations of this scale like any subjective semiquantitative scale which attempts to provide a holistic and clinical assessment of the complex aetiology of sleep problems in Parkinson's disease.

First, we have not validated this instrument against a gold standard measurement of sleep architecture such as polysomnography. However, we feel that a complete validation of the PDSS is impossible, as several of the 15 items have no gold standards that could be validated polysomnographically. Our aim is to provide a simple, clinical, inexpensive bedside tool for semiquantitative evaluation of sleep problems in Parkinsonian syndromes.

Second, we are unable to comment on the confounding impact of concomitant medical conditions upon the PDSS scores obtained for individual items. The age matched controls, however, may have suffered from a similar amount of concomitant disorders. It would be necessary to control for depression, psychosis, and other disorders such as arthritis that may have a confounding influence on sleep in future studies.

\section{Conclusions}

We suggest the PDSS be used as a simple bedside screening tool for identifying sleep problems in Parkinson's disease. We believe that patients scoring poorly on certain items such as 1 , 3, 14, and 15 may merit referral for formal laboratory based sleep studies and measurement of sleep architecture. By identifying individual symptoms contributing to disturbed sleep, the PDSS provides an objective method for targeted therapeutic approaches for the treatment of nocturnal symptoms in Parkinson's disease.

\section{ACKNOWLEDGEMENTS}

We acknowledge the support of Pharmacia and Dr Bruno Musch in creating the database and providing travelling expenses for SP and FRP to present data related to PDSS at several international meetings. We also acknowledge the help of Dr Linda Appiah-Kubi with the database and comments in relation to methodology; Dr Nirmala Mathew, Swapna Mandal, and C Lomax for collecting control data; and Dr Chris Clough for providing patients for the study. KRC is indebted to Professor J D Parkes for advise regarding initial development of sleep scale question items.

\section{Authors' affiliations}

K R Chaudhuri, S Pal, Department of Neurology, King's College

Hospital, London SE5, UK

A Forbes, University Hospital Lewisham, London, UK

A DiMarco, R Mathew, Guy's, King's and St Thomas' School of

Medicine, King's College, London, UK

C Trenkwalder, Department of Clinical Neurophysiology, University of

Göttingen, Germany

F R Pezzela, Department of Neurological Science, University of Rome La Sapienza, Rome, Italy

K Bridgman, Pharmacia Limited, Milton Keynes, UK

B Högl, Max-Planck Institute of Psychiatry, Munich, Germany

C Whately-Smith, Independent statistician

Competing interests: C W-S has previously undertaken statistical analysis and consulting work for Pharmacia Ltd. CT has used the PDSS scale within a treatment study has been supported by a research grant of Pharmacia. KB is an employee of Pharmacia Ltd. KRC has received honorarium from Pharmacia and Orion pharmaceuticals for presentation of data related to PDSS in the UK and abroad.

\section{REFERENCES}

1 Lees AJ, Blackburn, NA, Campbell VL. The nighttime problems of Parkinson's disease. Clin Neuropharmacol 1988;6:512-19.

2 Askenasy JJM. Sleep in Parkinson's disease. Acta Neurol Scand 1993;87: 167-70. 
3 Rye DB, Jankovic J. Emerging views of dopamine in modulating sleep/wake state from an unlikely source: Parkinson's disease. Neurology 2002;58:341-6.

4 Lang AE, Hobson DE, Martin W, et al. Excessive daytime sleepiness and sudden onset sleep in Parkinson's disease: a survey from 18 Canadian Movement Disorders Clinics [abstract]. Neurology 2001;56/suppl 3):A307.

5 Kales A, Ansel RD, Markham CH, et al. Sleep in patients with Parkinson's disease and normal subjects prior to and following levodopa administration. Clin Pharm Ther 1971:12:397-406.

6 Schenck CH, Bundlie SR, Mahowald MW. Delayed emergence of a parkinsonian disorder in $38 \%$ of 29 older men initially diagnosed with idiopathic rapid eye movement sleep behaviour disorder. Neurology 1996:46:388-93.

7 Ray Chaudhuri K, Pal S, Bridgman K, et al. Achieving 24-hr control of Parkinson's disease symptoms; use of objective measures to improve nocturnal disability. Eur Neurol $2001 ; 46$ (S1):3-10.

8 Reuter I, Ray Chaudhuri K. The management of nocturnal disabilities in Parkinson's disease. In: Tod D, ed. NAFP official yearbook. London: Scorpio Publishing, 1998:311-14.

9 Johns MW. A new method for measuring daytime sleepiness: the Epworth Sleepiness Scale. Sleep 1991;14:540-5.

10 Arnulf $I$, Konofal E, Merino-Andreau $M$, et al. Is excessive daytime sleepiness in Parkinson's disease related to poor sleep quality? An objective study of 47 patients [abstract]. Neurology 2001;56/suppl 3):A307.

11 Fahn S, Elton RL, Members of the UPDRS Development Committee. Unified Parkinson"s disease rating scale. In: Fahn S, Marsden CD, Calne $D$, et al, eds. Recent developments in Parkinson's disease. Florham Park: Macmillan Healthcare Information, 1987:153-63.

12 Ray Chaudhuri K, Pal S, Trenkwalder C, et al. Development of a novel scale for assessment of nocturnal disability in Parkinson's disease. $J$ Neurol Neurosurg Psychiatry 2001;70:281.

13 Addington-Hall J, Kalra L. Who should measure quality of life? BM 2001;322:1417-20.

14 Fayers PM, Machin D. Quality of life: assessment. analysis and interpretation. New York: John Wiley, 2000.

15 Holm S. A simple sequentially rejective Bonferri test procedure. Scand J Stat 1979:6:65-70.

16 Tan EK, Lum SY, Fook Chong SMC, et al. Evaluation of somnolence in Parkinson's disease: comparison with age and sex-matched controls. Neurology 2002;3:465-8.

17 Arnulf I, Konofal E, Merino-Andreu M, et al. Parkinson's disease and sleepiness: an integral part of PD. Neurology 2002;58:1019-24.

18 Pal S, DiMarco A, Prezzella FR, et al. Identification of a syndrome of nocturnal restlessness in Parkinson's disease using a novel visual analogue sleep scale. J Neurol Neurosurg Psychiatry (in press).
19 Ray Chaudhuri K, Bhattacharya K, Agapito C, et al. The use of cabergoline in nocturnal parkinsonian disabilities causing sleep disruption: a parallel study with controlled release levodopa. Eur J Neurol 1999;6(suppl 5):S11-15.

20 Marsden CD. Clinical experience with cabergoline in patients with advanced Parkinson's disease treated with levodopa. Drugs 1998;55(suppl 1):17-22.

21 Reuter I, Ellis CM, Ray Chaudhuri K. Nocturnal subcutaneous apomorphine infusion in Parkinson's disease and restless legs syndrome. Acta Neurol Scand 1999;100:163-7.

22 Ghatani T, Agapito C, Bhattacharya K, et al. Comparative audit of pergolide and cabergoline therapy in the treatment of nocturnal "off" periods causing sleep disruption in Parkinson"s disease. Eur J Neurol 2001;8(suppl 1):8-11.

23 Van der Kechove, Jacquy J, Garce M, et al. Sustained-release levodopa in parkinsonian patients with nocturnal disturbances. Acta Neurol Belg 1993;93:32-9.

24 Hogl B, Rothdach A, Wetter TC, et al. Effects of cabergoline on sleep and morning motor performance in patients with Parkinson's disease. Eur J Neurol 1999;6 (suppl 5):S25-8.

25 Arnulf I, Bejiani BP, Garma L, et al. Improvement of sleep architecture with subthalamic nucleus stimulation. Neurology 2001;55:1732-4.

26 Tandberg E, Larsen JP, Karlsen K. Excessive daytime sleepiness and sleep benefit in Parkinson's disease: a community based study. Mov Disord 1999;14:922-7.

27 Ondo WG, Voung KV, Khan H, et al. Daytime sleepiness and other sleep disorders in Parkinson"s disease. Neurology 2001;57:1392-6.

28 Frucht S, Rogers JD, Greene PE, et al. Falling asleep at the wheel: motor vehicle mishaps in persons taking pramipexole and ropinirole. Neurology 1999;52:1908-10.

29 Rye DB, Bliwise DL, Dihenia B, et al. Fast track: daytime sleepiness and other sleep disorders in Parkinson's disease. J Sleep Res 2000;9:63-9.

30 Rascol O, Ferreira JJ, Desboeuf K, et al. Sleep disruption, daytime somnolence, and "sleep attacks" in Parkinson's disease: a clinical survey in PD patients and age-matched healthy volunteers [abstract]. Neurology 2001;56(suppl 3):A212.

31 Kryger MH, Roth, T, Dement WC. Daytime sleepiness and alertness. In: Roth T, Roehrs TA, Carskadon MA, et al, eds. Principles and practice of sleep medicine. Philadelphia: WB Saunders, 1994:40-9.

32 Ray Chaudhuri K, Pal S, Forbes A, et al. Does nocturnal sleep disturbance contribute to excessive daytime sleepiness in Parkinson's disease [abstract]. J Neurol Sci 2001;S395:P1 162.

33 Stepanski J, Lamphere J, Badia $P$, et al. Sleep fragmentation and daytime sleepiness. Sleep 1984;7:18-26. 